\title{
Prevalence of mild cognitive impairment in employable patients after acute coronary event in cardiac rehabilitation
}

This article was published in the following Dove Press journal:

Vascular Health and Risk Management

23 February 2017

Number of times this article has been viewed

\author{
Annett Salzwedel' \\ Maria-Dorothea Heidler ${ }^{1,2}$ \\ Kathrin Haubold' \\ Martin Schikora ${ }^{2}$ \\ Rona Reibis ${ }^{3}$ \\ Karl Wegscheider ${ }^{4}$ \\ Michael Jöbges ${ }^{2}$ \\ Heinz Völler ${ }^{1,5}$ \\ 'Center for Rehabilitation Research, \\ University of Potsdam, Potsdam, \\ ${ }^{2}$ Brandenburg Klinik, Bernau, \\ ${ }^{3}$ Cardiological Outpatient Clinic, Am \\ Park Sanssouci, Potsdam, ${ }^{4}$ Institute for \\ Medical Biometry and Epidemiology, \\ University Medical Center Hamburg- \\ Eppendorf, Hamburg, ${ }^{5}$ Klinik am See, \\ Rüdersdorf, Germany
}

Introduction: Adequate cognitive function in patients is a prerequisite for successful implementation of patient education and lifestyle coping in comprehensive cardiac rehabilitation (CR) programs. Although the association between cardiovascular diseases and cognitive impairments (CIs) is well known, the prevalence particularly of mild CI in CR and the characteristics of affected patients have been insufficiently investigated so far.

Methods: In this prospective observational study, 496 patients ( $54.5 \pm 6.2$ years, $79.8 \%$ men) with coronary artery disease following an acute coronary event (ACE) were analyzed. Patients were enrolled within 14 days of discharge from the hospital in a 3-week inpatient CR program. Patients were tested for CI using the Montreal Cognitive Assessment (MoCA) upon admission to and discharge from CR. Additionally, sociodemographic, clinical, and physiological variables were documented. The data were analyzed descriptively and in a multivariate stepwise backward elimination regression model with respect to $\mathrm{CI}$.

Results: At admission to CR, the CI (MoCA score < 26) was determined in 182 patients (36.7\%). Significant differences between CI and no CI groups were identified, and CI group was associated with high prevalence of smoking ( 65.9 vs $56.7 \%, P=0.046)$, heavy (physically demanding) workloads ( 26.4 vs $17.8 \%, P<0.001)$, sick leave longer than 1 month prior to $\mathrm{CR}$ (28.6 vs $18.5 \%, P=0.026)$, reduced exercise capacity ( 102.5 vs $118.8 \mathrm{~W}, P=0.006)$, and a shorter 6-min walking distance ( 401.7 vs $421.3 \mathrm{~m}, P=0.021$ ) compared to no CI group. The age- and education-adjusted model showed positive associations with CI only for sick leave more than 1 month prior to ACE (odds ratio [OR] 1.673, 95\% confidence interval 1.07-2.79; $P=0.03$ ) and heavy workloads (OR 2.18, 95\% confidence interval $1.42-3.36 ; P<0.01$ ).

Conclusion: The prevalence of $\mathrm{CI}$ in $\mathrm{CR}$ was considerably high, affecting more than one-third of cardiac patients. Besides age and education level, CI was associated with heavy workloads and a longer sick leave before ACE.

Keywords: cardiac rehabilitation, cognitive impairment, prevalence, acute coronary event, patient education

\section{Introduction}

For patients with coronary artery disease (CAD), especially following an acute coronary event (ACE) such as myocardial infarction or coronary artery bypass grafting (CABG), patient education is a core component of multicomponent cardiac rehabilitation (CR).

The CR education program for patients with CAD in Germany is based on a standardized curriculum. It includes aspects of secondary prevention: cardiovascular function disorders, symptoms of angina pectoris and myocardial infarction, cardiovascular risk factors, treatment of acute coronary syndrome (ACS), medical and therapeutic treatment of CAD, handling of CAD in everyday life, and necessary lifestyle changes. ${ }^{1}$ For a suc-
Correspondence: Annett Salzwedel Center for Rehabilitation Research, University of Potsdam, Am Neuen Palais 10, 14469 Potsdam, Germany Tel +49331977406 I Fax +49 331 9774081 Email annett.salzwedel@uni-potsdam.de 
cessful implementation of this intensive education program, adequate cognitive function (e.g. executive function, selective attention, memory) is an important prerequisite.

Nevertheless, a substantial proportion of patients with cardiovascular diseases suffer from mild cognitive impairment (MCI). Newman et al reported cognitive dysfunctions in up to $53 \%$ of patients after coronary artery bypass surgery upon discharge from the hospital, decreasing to $24 \%$ after 6 months. ${ }^{2}$ MCI was found in survivors of acute myocardial infarction at $30 \%$, and moderate/severe cognitive impairment (CI) in $26 \%$ of patients within 1 month of the index event. ${ }^{3}$ Another study reported MCI in $17 \%$ of non-demented patients with coronary heart disease. ${ }^{4}$

Although the association of coronary heart disease with CIs is well documented particularly in older patients $\geq 65$ years, the prevalence of $\mathrm{CI}$ and its characteristics in a younger, employable patient population remain poorly investigated. Thus, we aimed to identify the prevalence of $\mathrm{CI}$ in CAD patients under 65 years undergoing $\mathrm{CR}$ following an acute event. We hypothesized a prevalence rate of at least $25 \%$. In addition, we focused on associations between CI and sociodemographic, clinical, and physiological variables.

\section{Methods}

\section{Patients}

From September 2014 to August 2015, a bicentric, prospective observational study was carried out within a 3-week inpatient CR program. Upon admission to CR, all eligible patients were screened for inclusion criteria: the allocation to $\mathrm{CR}$ had to be due to an ACE. Additionally, patients must have had a CAD diagnosis, they had to be between 18 and 65 years old, and insured by a German health insurance company. Patients with insufficient language skills, those who declined to give informed consent, and those diagnosed with dementia were excluded. From 647 eligible patients, 520 were included in the study, of which 24 dropped out due to rehospitalization, withdrawal, or organizational issues. Thus, 496 consecutive Caucasian patients were included in the analysis.

\section{CR}

All patients underwent an intensive, standardized, multicomponent CR program, as described elsewhere, ${ }^{5,6}$ for a mean period of 21 days, starting, on average, 14 days after discharge from the hospital following an ACE. In addition to medical care, the program primarily included exercise training, psychological care (individual counseling by a psychologist and/or group sessions for coping), nutritional advice, and patient education for the secondary prevention of CAD including cardiovascular function disorders and risk factors, treatment of CAD, handling of CAD in everyday life, and necessary lifestyle changes. ${ }^{1}$

\section{Cognitive performance measurement and data collection}

All patients were tested for CI using the Montreal Cognitive Assessment (MoCA) with less than 26 points as the cutoff for CI. ${ }^{7}$ To avoid learning effects, two German versions of the MoCA were executed by qualified and trained study personnel upon admission to and discharge from CR. Where the original version from November $7,2004^{8}$ was used at admission, the alternate version from $2012^{9}$ was performed upon discharge from $\mathrm{CR}$.

Besides cognitive function, sociodemographic, clinical, and physiological variables (e.g. age, sex, education level, employment status, light or heavy [physically demanding] workloads, cardiovascular risk factors, comorbidities, New York Heart Association [NYHA] class, left ventricular ejection fraction [LVEF], extent of depression and anxiety in the Hospital Anxiety and Depression Scale [HADS], 6-min walking distance [6MWD], and maximum capacity in the exercise stress test) were documented.

Data were collected in two inpatient CR centers at admission to and discharge from CR. The information was prospectively compiled by study nurses, whereby clinical and anamnestic data were taken from the patient's chart in the rehabilitation center. Due to unperformed assessments in the CR routine, missing data were observed for the 6MWD $(n=18)$, the exercise stress test $(n=23)$, and HADS $(n=39)$. For the other variables, all records were complete.

\section{Statistics}

Continuous variables are expressed as mean \pm standard deviation, and categorical variables as frequencies and percentages. Comparisons between CI cases and patients with normal cognitive function were made using a chi-squared test for categorical variables or a Students $t$-test for continuous variables.

To determine associations between patient characteristics and CI, an exploratory, multivariate, binary logistic regression model with stepwise backward elimination was applied. Variables known to associate with cognitive decline ${ }^{10-12}$ and sociodemographic and clinical variables found to be significant in bivariate analysis $(P \leq 0.1)$ were considered in the model: age, education level, sick leave before ACE, heavy workload, HADS, psychological comorbidity, chronic obstructive pulmonary disease, number of comorbidities, smoking, diabetes mellitus, arterial hypertension, number of cardiovascular risk factors, medication (tranquilizers, 
antidepressants, anticonvulsants, or neuroleptics), reduced LVEF, and therapy of ACEs (percutaneous coronary intervention $[\mathrm{PCI}]$ or $\mathrm{CABG})$. Effects with a $P$-value of less than 0.05 were considered statistically significant. The effect-size estimates (odds ratios, ORs) of the independent predictor variables for the final model are shown with 95\% confidence intervals and $P$-values. Multicollinearity was assessed through variance inflation factor (VIF). Calculations were carried out using Statistical Package for the Social Sciences (SPSS) 23.0 (IBM, Armonk, NY, USA).

\section{Ethics}

All patients were informed and educated about the study realities and gave written informed consent prior to enrollment. The study was approved by the ethics committee of the Human Sciences Faculty of the University of Potsdam in Germany and was registered at the German Clinical Trial Registry and the International Clinical Trials Registry Platform (ICTRP) of the World Health Organization (DRKS00005502).

\section{Results}

The mean age of the patients was $54.5 \pm 6.2$ years, $79.8 \%$ were men, and $81.0 \%$ had an educational level beyond high school. CI was determined in 182 patients (36.7\%) upon admission to CR, whereof 173 cases (95.1\%) could be classified as MCI. The mean scores of MoCA in the CI group were 22.74 \pm 2.32 , while MoCA scores of patients without CI were $27.89 \pm 1.51$ (Table 1). Upon discharge from CR, the rate of CI was significantly reduced to $32.9 \%(\mathrm{n}=163 ; P<0.001)$. The CI group achieved on average $23.06 \pm 2.14$ points in the MoCA, while $28.08 \pm 1.41$ points were scored by the no CI group (Table 1 ). MoCA scores beyond the lower normative data range for MCI $(<19)$ were found at admission and discharge from CR in nine patients (4.9\%) and were statistically classified as outliers.

Significant differences between CI and no CI groups at admission were identified, and CI group was associated with high prevalence of smoking ( 65.9 vs $56.7 \%, P=0.046)$, heavy

Table I Cognitive performance at admission to and discharge from CR

\begin{tabular}{llll}
\hline & $\begin{array}{l}\text { Cl (MoCA } \\
\text { < 26) }\end{array}$ & $\begin{array}{l}\text { No Cl (MoCA } \\
\mathbf{2 6} \text { ) }\end{array}$ & Total \\
\hline Admission to CR, $\mathrm{n}$ & 182 & 314 & \\
MoCA scores, mean \pm & $22.74 \pm 2.32$ & $27.89 \pm 1.5 I$ & $26.00 \pm 3.10$ \\
SD (range) & $(12-25)$ & $(26-3 \mid)$ & \\
Discharge from CR, $\mathrm{n}$ & 163 & 333 & \\
MoCA scores, mean \pm & $23.06 \pm 2.14$ & $28.08 \pm 1.4 \mathrm{I}$ & $26.42 \pm 2.9 \mathrm{I}$ \\
SD (range) & $(15-25)$ & $(26-3 \mathrm{I})$ & \\
\hline
\end{tabular}

Abbreviations: $\mathrm{CR}$, cardiac rehabilitation; $\mathrm{Cl}$, cognitive impairment; MoCA, Montreal Cognitive Assessment; SD, standard deviation. workloads ( 26.4 vs $17.8 \%, P<0.001)$, sick leave longer than 1 month prior to CR (28.6 vs $18.5 \%, P=0.026$ ), reduced exercise capacity ( 102.5 vs $118.8 \mathrm{~W}, P=0.006)$, and a shorter 6MWD (401.7 vs $421.3 \mathrm{~m}, P=0.021$ ). No differences were found for sex, living situation, alcohol consumption, comorbidities, cardiovascular risk factors, psychotropic medication, NYHA class, peri-interventional ACS therapy (CABG vs PCI), LVEF, or depression and anxiety scores (Table 2).

The multivariate analysis showed only four statistically significant associations in the final step: besides age (OR 1.054 per year, 95\% confidence interval 1.02-1.09; $P=0.005$ ) and education level (graduation after 10 years of school vs lower level or no graduation: OR $0.221,95 \%$ confidence interval $0.123-0.396 ; P<0.001$ ), only sick leave longer than 1 month before ACE (OR 1.673, 95\% confidence interval 1.07-2.79; $P=0.03$ ) and heavy workloads (OR 2.18, $95 \%$ confidence interval $1.42-3.36 ; P<0.01)$ could be determined as independent predictors of $\mathrm{CI}$ at admission to CR. No strong collinearity was found between the variables analyzed in the model, and VIF ranged from 1.011 for sick leave longer than 1 month before ACE to 1.098 for educational level. Patients with MoCA scores under 19 points (severe CI) had no appreciable influence on the multivariate analysis and therefore were not excluded or separately grouped.

\section{Discussion}

The prevalence of $\mathrm{CI}$ at admission to $\mathrm{CR}$ was considerably high and was detected in $36.7 \%$ of the comparatively young CAD patients after an ACE. However, as majority of patients scored in the MoCA within the normative data range for MCI (95\%) and the fact that the small ratio of patients with more severe CI (5\%) showed no influences on the statistical calculations, the $\mathrm{CI}$ in this population could be described as MCI. While the prevalence of MCI in the regular Caucasian population within the $60-65$ age group is estimated to be $11-17 \%,{ }^{13}$ previous studies in cardiac patients showed a comparable or even higher prevalence of $\mathrm{MCI},{ }^{2-4}$ although the patients enrolled at that time were older than our population.

Certainly, CI is predominantly detected through test procedures, ${ }^{14}$ and its prevalence would be presumably higher and more precise if a standardized neuropsychological test battery is used. ${ }^{15}$ But, even using a simple screening tool like the MoCA, we were able to demonstrate a high CI prevalence in our heterogeneous sample of CAD patients. Nonetheless, MoCA is a screening tool with a high degree of sensitivity even for patients with cardiovascular disease. ${ }^{7}$ However, the observed mean MoCA scores and standard deviations in the $\mathrm{CI}$ and no CI groups were comparable to the normative data 
Table 2 Patient characteristics: total and by cognitive status at admission to cardiac rehabilitation

\begin{tabular}{|c|c|c|c|c|}
\hline Patient characteristics & Total $(N=496)$ & $\begin{array}{l}\text { Normal cognitive } \\
\text { function }(N=314)\end{array}$ & $\begin{array}{l}\text { Cognitive impairment } \\
(N=182)\end{array}$ & $P$-value \\
\hline \multicolumn{5}{|l|}{ Sociodemographic parameters } \\
\hline Age (years) & $54.5 \pm 6.2$ & $53.9 \pm 6.5$ & $55.5 \pm 5.5$ & 0.005 \\
\hline Sex & & & & 0.298 \\
\hline Women & $100(20.2)$ & $68(2 \mid .7)$ & $32(17.6)$ & \\
\hline Men & $396(79.8)$ & $246(78.3)$ & $150(82.4)$ & \\
\hline BMI $\left(\mathrm{kg} / \mathrm{m}^{2}\right)$ & $28.7 \pm 5.1$ & $28.6 \pm 5.1$ & $29.0 \pm 5.2$ & 0.361 \\
\hline Living alone & $103(20.8)$ & $59(18.8)$ & $44(24.2)$ & 0.169 \\
\hline Graduation & & & & $<0.001$ \\
\hline Education $<10$ years & $78(15.7)$ & $24(7.6)$ & $54(29.7)$ & \\
\hline Education 10 years & $324(65.3)$ & $216(68.8)$ & $108(59.3)$ & \\
\hline Higher education & $94(19.0)$ & $74(23.6)$ & $20(I I .0)$ & \\
\hline Associate degree & & & & 0.019 \\
\hline No degree & $27(5.4)$ & $13(4.1)$ & $14(7.7)$ & \\
\hline Vocational education & $398(80.2)$ & $247(78.7)$ & $|5|(83.0)$ & \\
\hline University degree & $71(14.3)$ & $54(17.2)$ & $17(9.3)$ & \\
\hline Incapacity for work prior to ACS (months) & $1.5 \pm 3.0$ & $1.3 \pm 2.9$ & $1.8 \pm 3.4$ & 0.085 \\
\hline Employed prior to ACS & $406(81.9)$ & $267(85.0)$ & $139(76.4)$ & 0.021 \\
\hline Heavy work & $104(21.0)$ & $56(17.8)$ & $48(26.4)$ & $<0.001$ \\
\hline ACS treatment & & & & 0.497 \\
\hline $\mathrm{PCl}$ & $335(67.5)$ & $213(67.8)$ & $122(67.4)$ & \\
\hline CABG & $140(28.2)$ & $86(27.4)$ & $54(29.8)$ & \\
\hline Conservative procedure & $20(4.0)$ & $15(4.8)$ & $5(2.8)$ & \\
\hline \multicolumn{5}{|l|}{ Cardiovascular risk factors } \\
\hline Hypertension & $34 \mid(68.8)$ & $219(69.7)$ & $122(67.0)$ & 0.547 \\
\hline Diabetes mellitus & $109(22.0)$ & $63(20.1)$ & $46(25.3)$ & 0.216 \\
\hline Dyslipidemia & $319(64.3)$ & $194(6 \mid .8)$ & $125(68.7)$ & 0.145 \\
\hline Smoker/ex-smoker (<12 months) & $298(60.1)$ & $178(56.7)$ & $120(65.9)$ & 0.046 \\
\hline Number of risk factors & $2.2 \pm 0.9$ & $2.1 \pm 0.9$ & $2.3 \pm 0.9$ & 0.039 \\
\hline \multicolumn{5}{|l|}{ Comorbidities } \\
\hline Mental disease & $54(10.9)$ & $28(8.9)$ & $26(14.3)$ & 0.073 \\
\hline COPD & $37(7.5)$ & $18(5.7)$ & $19(10.4)$ & 0.075 \\
\hline Stroke & $17(3.4)$ & II (3.5) & $6(3.3)$ & 1.000 \\
\hline \multicolumn{5}{|l|}{ Functional parameters } \\
\hline LVEF & $55.2 \pm 7.8$ & $55.5 \pm 7.8$ & $54.8 \pm 8.0$ & 0.365 \\
\hline Reduced LVEF (<45\%) & $78(15.7)$ & $45(14.3)$ & $33(18.1)$ & 0.306 \\
\hline 6-min walking distance $(\mathrm{m})$ & $4 \mid 4.2 \pm 88.8$ & $421.3 \pm 84.3$ & $401.7 \pm 95.2$ & 0.021 \\
\hline Exercise capacity $(\mathrm{W})^{\mathrm{a}}$ & $108.4 \pm 36.3$ & $111.8 \pm 34.9$ & $102.5 \pm 38.0$ & 0.006 \\
\hline \multicolumn{5}{|l|}{ Psychological screening (HADS) } \\
\hline Depression & $5.3 \pm 3.9$ & $5.2 \pm 4.0$ & $5.6 \pm 3.8$ & 0.328 \\
\hline Anxiety & $6.3 \pm 4.1$ & $6.4 \pm 4.0$ & $6.4 \pm 4.4$ & 0.686 \\
\hline
\end{tabular}

Notes: Results are presented as mean \pm standard deviation or number of subjects (percentage). HADS scoring for each subscale (anxiety and depression) is as follows:

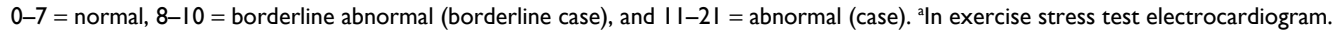

Abbreviations: BMI, body mass index; ACS, acute coronary syndrome; PCl, percutaneous coronary intervention; CABG, coronary artery bypass grafting; COPD, chronic obstructive pulmonary disease; LVEF, left ventricular ejection fraction; HADS, Hospital Anxiety and Depression Scale.

for the MoCA (MCI 22.1 \pm 3.1 and non-CI $27.4 \pm 2.2$ ). ${ }^{8}$ This underlines the assumption that our examined population on average corresponds to an MCI group.

Interestingly, CI was associated with sick leave before ACE and heavy workloads, while clinical conditions did not seem to be relevant. There is still conflicting evidence regarding the impact of LVEF on cognitive function. . $^{12,16,17}$ In contrast to other authors, we did not find an association between $\mathrm{CI}$ and reduced LVEF. ${ }^{18-20}$ However, this association was reported mainly for patients with reduced LVEF $(\leq 40 \%)$, which applied only to 38 patients in our sample (mean LVEF $55.2 \pm 7.8 \%$ ). However, only a weak correlation between LVEF, other clinical conditions (such as NYHA class), and cognitive function has been shown in other earlier studies as well. ${ }^{12,21}$

CIs may be promoted by an adverse lifestyle (e.g. smoking, alcohol abuse, or unhealthy nutrition ${ }^{22,23}$ and a reduced adherence to treatment. ${ }^{24}$ Multivariable analysis of the present 
study showed positive associations with CI for age, physically demanding work (e.g. in professions such as commercial drivers, building workers, or geriatric nurses), and education (even after adding a point for less than 12 years of formal education in the MoCA). ${ }^{9,25}$ This prevailing association of psychosocial rather than clinical factors with $\mathrm{CI}$ could be an indication of a potential reverse causation: it can be hypothesized that cognitive deficits were preexisting, serving as a cofactor in the development of CAD and ACS. ${ }^{26}$ In turn, ACS could aggravate $\mathrm{CI}$ due to regional brain changes, which may explain the notable prevalence rate of CI in the given population. ${ }^{27,28}$

Apart from this, the influence of educational level should be discussed. The assessment instructions for MoCA provide an additional point for education lasting less than 12 years. However, our study findings emphasize a strong influence of formal education lasting less than 10 years according to several studies confirming the influence of different educational levels. ${ }^{29,30}$ It is advisable to conduct a validation study of the German MoCA versions with special consideration given to cardiac patients' educational levels.

Moreover, it is hypothesized that cognitive decline depends on the presence of several cerebrovascular risk factors and cardiovascular comorbidities; many manifestations of cardiovascular disease (such as hypertension or atherosclerosis) coexist within the same patient and therefore cannot be viewed in isolation. ${ }^{31,32}$ Furthermore, cerebrovascular disease has been suggested as an underlying pathophysiology of both cardiac disease and cognitive decline. ${ }^{33}$ So, it is hardly surprising that we could not find specific parameters for CI assuming a multifactorial etiology and the same underlying mechanisms for $\mathrm{CI}$ and cardiovascular disease such as CAD.

Our study has several limitations. First, a single score from a screening instrument was used to operationalize cognitive functioning and impairment (although MoCA is a screening tool with high sensitivity). Second, there was no control group to rate $\mathrm{CI}$ in age- and sex-matched individuals without ACS, so we referred to the literature data. ${ }^{71,34-36}$ Third, no information regarding possibly preexisting cognitive deficits in patients which might be linked to cardiovascular risk factors was available. Studies show that preexisting CIs are significantly linked to postoperative cognitive deficits following cardiac surgery. ${ }^{37}$ In comparison with healthy controls, previous trials demonstrated lower performances on all cognitive tests by patients scheduled for coronary angiography, cardiac surgery, or CABG. ${ }^{38-40}$ Additionally, we did not have any data regarding the severity of peri-infarctional hemodynamic instability and possible hypoxic brain injury which could contribute to decreased cognitive function in our patients. ${ }^{41}$
Further investigations are required to clarify the impact of CI on patients' CR outcome, especially the implementation of healthy lifestyle and treatment compliance as an index of sustained education success through multicomponent training.

\section{Conclusion}

Even if the results cannot be generalized due to several study limitations, the prevalence of CI in relatively young employable patients with ACS in the present study was considerably high. CIs should be taken into account when planning and implementing CR programs.

\section{Acknowledgments}

The authors acknowledge the support of the Deutsche Forschungsgemeinschaft and Open Access Publishing Fund of University of Potsdam. This work was funded by the German Federal Pension Insurance (grant no. 0421/00-40-64-50-39).

\section{Disclosure}

The authors report no conflicts of interest in this work.

\section{References}

1. Seekatz B, Haug G, Mosler G, et al. Entwicklung und kurzfristige Effektivität eines standardisierten Schulungsprogramms für die Rehabilitation bei koronarer Herzkrankheit [Development and short-term effects of a standardized patient education program for in-patient cardiologic rehabilitation]. Rehabilitation (Stuttg). 2013;52(5):344-351. German [with English abstract].

2. Newman MF, Grocott HP, Mathew JP, et al; Neurologic Outcome Research Group and the Cardiothoracic Anesthesia Research Endeavors (CARE) Investigators of the Duke Heart Center. Report of the substudy assessing the impact of neurocognitive function on quality of life 5 years after cardiac surgery. Stroke. 2001;32(12):2874-2881.

3. Gharacholou SM, Reid KJ, Arnold SV, et al. Cognitive impairment and outcomes in older adult survivors of acute myocardial infarction: findings from the translational research investigating underlying disparities in acute myocardial infarction patients' health status registry. $\mathrm{Am}$ Heart J. 2011;162(5):860-869.e1.

4. Roberts RO, Knopman DS, Geda YE, Cha RH, Roger VL, Petersen RC. Coronary heart disease is associated with non-amnestic mild cognitive impairment. Neurobiol Aging. 2010;31(11):1894-1902.

5. Rauch B, Riemer T, Schwaab B, et al; OMEGA study group. Shortterm comprehensive cardiac rehabilitation after AMI is associated with reduced 1-year mortality: results from the OMEGA study. Eur J Prev Cardiol. 2014;21(9):1060-1069.

6. Karoff M, Held K, Bjarnason-Wehrens B. Cardiac rehabilitation in Germany. Eur J Cardiovasc Prev Rehabil. 2007;14(1):18-27.

7. McLennan SN, Mathias JL, Brennan LC, Stewart S. Validity of the Montreal Cognitive Assessment (MoCA) as a screening test for mild cognitive impairment (MCI) in a cardiovascular population. $J$ Geriatr Psychiatry Neurol. 2011;24(1):33-38.

8. Nasreddine ZS, Phillips NA, Bédirian V, et al. The Montreal Cognitive Assessment, MoCA: a brief screening tool for mild cognitive impairment. J Am Geriatr Soc. 2005;53(4):695-699.

9. Costa AS, Fimm B, Friesen P, et al. Alternate-form reliability of the Montreal cognitive assessment screening test in a clinical setting. Dement Geriatr Cogn Disord. 2012;33(6):379-384. 
10. Singh B, Mielke MM, Parsaik AK, et al. A prospective study of chronic obstructive pulmonary disease and the risk of mild cognitive impairment. JAMA Neurol. 2014;71(5):581-588.

11. Li W, Wang T, Xiao S. Type 2 diabetes mellitus might be a risk factor for mild cognitive impairment progressing to Alzheimer's disease. Neuropsychiatr Dis Treat. 2016;12:2489-2495.

12. Burkauskas J, Brozaitiene J, Bunevicius A, Neverauskas J, Zaliunaite V, Bunevicius R. Association of depression, anxiety, and type $\mathrm{D}$ personality with cognitive function in patients with coronary artery disease. Cogn Behav Neurol. 2016;29(2):91-99.

13. Mariani E, Monastero R, Mecocci P. Mild cognitive impairment: a systematic review. J Alzheimers Dis. 2007;12(1):23-35.

14. Pendlebury ST, Mariz J, Bull L, Mehta Z, Rothwell PM. MoCA, ACE$\mathrm{R}$, and MMSE versus the National Institute of Neurological Disorders and Stroke-Canadian Stroke Network Vascular Cognitive Impairment Harmonization Standards Neuropsychological Battery after TIA and stroke. Stroke. 2012;43(2):464-469.

15. Diniz BS, Nunes PV, Yassuda MS, et al. Mild cognitive impairment: cognitive screening or neuropsychological assessment? Rev Bras Psiquiatr. 2008;30(4):316-321.

16. Arangalage D, Ederhy S, Dufour L, et al. Relationship between cognitive impairment and echocardiographic parameters: a review. J Am Soc Echocardiogr. 2015;28(3):264-274.

17. Bennett SJ, Sauvé MJ. Cognitive deficits in patients with heart failure: a review of the literature. J Cardiovasc Nurs. 2003;18(3):219-242.

18. Hoth KF, Poppas A, Moser DJ, Paul RH, Cohen RA. Cardiac dysfunction and cognition in older adults with heart failure. Cogn Behav Neurol. 2008; 21(2):65-72.

19. Jerskey BA, Cohen RA, Jefferson AL, et al. Sustained attention is associated with left ventricular ejection fraction in older adults with heart disease. J Int Neuropsychol Soc. 2009;15(1):137-141.

20. Festa JR, Jia X, Cheung K, et al. Association of low ejection fraction with impaired verbal memory in older patients with heart failure. Arch Neurol. 2011;68(8):1021-1026.

21. Beer C, Ebenezer E, Fenner S, et al. Contributors to cognitive impairment in congestive heart failure: a pilot case-control study. Intern Med J. 2009;39(9):600-605.

22. Anstey KJ, Kingston A, Kiely KM, Luszcz MA, Mitchell P, Jagger C. The influence of smoking, sedentary lifestyle and obesity on cognitive impairment-free life expectancy. Int J Epidemiol. 2014;43(6):1874-1883.

23. Lee Y, Back JH, Kim J, et al. Systematic review of health behavioral risks and cognitive health in older adults. Int Psychogeriatr. 2010;22(2):174-187.

24. Alosco ML, Spitznagel MB, Cohen R, et al. Better adherence to treatment recommendations in heart failure predicts improved cognitive function at a one-year follow-up. J Clin Exp Neuropsychol. 2014; 36(9):956-966.

25. Tu QY, Jin H, Ding BR, et al. Reliability, validity, and optimal cutoff score of the Montreal cognitive assessment (Changsha version) in ischemic cerebrovascular disease patients of Hunan province, China. Dement Geriatr Cogn Dis Extra. 2013;3(1):25-36.
26. Cannon JA, McMurray JJ, Quinn TJ. 'Hearts and minds': association, causation and implication of cognitive impairment in heart failure. Alzheimers Res Ther. 2015;7(1):22.

27. Almeida OP, Garrido GJ, Beer C, et al. Coronary heart disease is associated with regional grey matter volume loss: implications for cognitive function and behaviour. Intern Med J. 2008;38(7):599-606.

28. Barekatain M, Askarpour H, Zahedian F, et al. The relationship between regional brain volumes and the extent of coronary artery disease in mild cognitive impairment. J Res Med Sci. 2014;19(8):739-745.

29. Freitas S, Simões MR, Alves L, Santana I. Montreal cognitive assessment: influence of sociodemographic and health variables. Arch Clin Neuropsychol. 2012;27(2):165-175.

30. Ng TP, Feng L, Lim WS, et al. Montreal cognitive assessment for screening mild cognitive impairment: variations in test performance and scores by education in Singapore. Dement Geriatr Cogn Disord. 2015; 39(3-4):176-185.

31. Weber MA, Bakris GL, Hester A, et al. Systolic blood pressure and cardiovascular outcomes during treatment of hypertension. Am J Med. 2013;126(6):501-508.

32. Asia Pacific Cohort Studies Collaboration. Joint effects of systolic blood pressure and serum cholesterol on cardiovascular disease in the Asia Pacific region. Circulation. 2005;112(22):3384-3390.

33. Stroobant N, Vingerhoets G. Pre-existing cognitive impairment in candidates for cardiac surgery: an overview. Heart. 2009;95(22): $1820-1825$

34. Bischkopf J, Busse A, Angermeyer MC. Mild cognitive impairment a review of prevalence, incidence and outcome according to current approaches. Acta Psychiatr Scand. 2002;106(6):403-414.

35. Dlugaj M, Weimar C, Wege N, et al; Heinz Nixdorf Recall Study Investigative Group. Prevalence of mild cognitive impairment and its subtypes in the Heinz Nixdorf recall study cohort. Dement Geriatr Cogn Disord. 2010;30(4):362-373.

36. Lopez OL, Jagust WJ, DeKosky ST, et al. Prevalence and classification of mild cognitive impairment in the Cardiovascular Health Study Cognition Study: part 1. Arch Neurol. 2003;60(10):1385-1389.

37. Millar K, Asbury AJ, Murray GD. Pre-existing cognitive impairment as a factor influencing outcome after cardiac surgery. Br JAnaesth. 2001; 86(1):63-67.

38. Vingerhoets G, Van Nooten G, Jannes C. Neuropsychological impairment in candidates for cardiac surgery. J Int Neuropsychol Soc. 1997; 3(5):480-484.

39. Silbert BS, Scott DA, Evered LA, Lewis MS, Maruff PT. Preexisting cognitive impairment in patients scheduled for elective coronary artery bypass graft surgery. Anesth Analg. 2007;104(5):1023-1028.

40. Evered LA, Silbert BS, Scott DA, et al. Plasma amyloid beta42 and amyloid beta40 levels are associated with early cognitive dysfunction after cardiac surgery. Ann Thorac Surg. 2009;88(5):1426-1432.

41. Hofmeijer J, Mulder AT, Farinha AC, van Putten MJ, le Feber J. Mild hypoxia affects synaptic connectivity in cultured neuronal networks. Brain Res. 2014;1557:180-189.
Vascular Health and Risk Management

\section{Publish your work in this journal}

Vascular Health and Risk Management is an international, peerreviewed journal of therapeutics and risk management, focusing on concise rapid reporting of clinical studies on the processes involved in the maintenance of vascular health; the monitoring, prevention and treatment of vascular disease and its sequelae; and the involvement of
Dovepress

metabolic disorders, particularly diabetes. This journal is indexed on PubMed Central and MedLine. The manuscript management system is completely online and includes a very quick and fair peer-review system, which is all easy to use. Visit http://www.dovepress.com/ testimonials.php to read real quotes from published authors. 